\title{
Exosomal microRNA-146a derived from mesenchymal stem cells increases the sensitivity of ovarian cancer cells to docetaxel and taxane via a LAMC2-mediated PI3K/Akt axis
}

\author{
LIYA QIU, JIAKUN WANG, MEI CHEN, FENGYUN CHEN and WENLUO TU
}

\author{
Department of Obstetrics and Gynecology, Taizhou Women and Children's Hospital, Taizhou, Zhejiang 318000, P.R. China
}

Received February 12, 2020; Accepted May 4, 2020

DOI: $10.3892 /$ ijmm.2020.4634

\begin{abstract}
The carrier role of exosomes from human umbilical cord mesenchymal stem cells (hUCMSCs) containing microRNAs (miRNAs) has been implicated in gene and drug therapy. The aim of the present study was to investigate the role of exosomal microRNA-146a (miR-146a) from hUCMSCs in ovarian cancer (OC). Following the generation of docetaxel (DTX)-resistant SKOV3 cells and taxane-resistant A2780 cells, exosomes were isolated from hUCMSCs and added to the chemoresistant cells. Microarray analysis revealed that miR-146a expression was upregulated in DTX/SKOV3 cells among 15 ectopically expressed miRNAs. Analysis using the StarBase and miRSearch databases demonstrated that miR-146a targeted laminin $\gamma 2$ (LAMC2), which was further verified using dual-luciferase reporter assays. Subsequently, miR-146a inhibitor or LAMC2 overexpression vectors were transfected into hUCMSCs or OC cells, respectively, and their effects on growth and chemoresistance in OC cells were assessed. The hUCMSC-derived exosomes reduced cell growth and chemoresistance in OC. Furthermore, hUCMSC-derived exosomes with miR-146a expression knocked down increased OC cell growth and chemoresistance, which was mediated by the PI3K/Akt signaling pathway via LAMC2.
\end{abstract}

\section{Introduction}

Ovarian cancer (OC) was the fifth most prevalent malignancy in females, and had the highest mortality rate among all types of gynecological cancer in the United States in 2015 (1). Conventional treatment strategies for OC include cytoreductive surgery accompanied by intermittent administration of chemotherapeutic agents, such as platinum or taxane (TAX),

Correspondence to: Mr. Wenluo Tu, Department of Obstetrics and Gynecology, Taizhou Women and Children's Hospital, 188 Qianjin Village, Jiahe Street, Jiaojiang, Taizhou, Zhejiang 318000, P.R. China E-mail: wenluotu121701@163.com

Key words: human umbilical cord mesenchymal stem cells, ovarian cancer, exosomes, microRNA-146a, laminin $\gamma 2$, chemoresistance at the maximum tolerated doses (2). Docetaxel (DTX) is a TAX which is a type of FDA-approved drug for use in OC, and is frequently used in a clinical setting (3). Despite significant research investments and efforts, the 5-year overall survival rate for patients with $\mathrm{OC}$ has only increased slightly since 1995 (4). Chemoresistance in OC is potentially caused by several mechanisms in a heterogeneous tumor cell population (5). Mesenchymal stem cells (MSCs) are a group of multipotent stromal cells which reside in several areas, such as the bone marrow, fat or the dental pulp (6). MSCs have been demonstrated to function in the tumor microenvironment, and are involved in tumor progression, metastasis and chemotherapeutic resistance (7). The human umbilical cord is a suitable source of MSCs, and unlike bone marrow-derived MSCs, human umbilical cord MSCs (hUCMSCs) are collected in a painless manner and exhibit faster self-renewal properties (8). MSCs potentially promote drug resistance either through direct contact with the tumor cells or via systemic mechanisms with the involvement of secreted factors (9). Exosomes, a subgroup of extracellular vesicles, may be released by virtually all mammalian cell types, and transfer a variety of molecules, including mRNAs and microRNAs (miRNAs/miRs), as well as long non-coding RNAs, any of which may confer resistance to drug-sensitive cancer cells (10).

miR-146a is an established anti-inflammatory miRNA and has been implicated as a therapeutic target for several inflammation-associated disorders, including peripartum cardiomyopathy (11) and obesity (12). Furthermore, hUCMSCs-derived exosomes have been reported to exert anti-inflammatory effects on human trophoblast cells by transferring miR-146a-5p (13). miR-146a has been demonstrated to serve a significant role in exosomes secreted by colorectal cancer stem cells, where exosomal miR-146a promotes stem-like properties and tumorigenicity (14). In addition, the oncogenic role of laminin $\gamma 2$ (LAMC2) in OC has been reported previously, and it increased p38 expression via miR-125a-5p (15). Therefore, it was hypothesized that exosomal miR-146a derived from hUCMSCs may affect chemoresistance to TAX and DTX by regulating LAMC2 in OC. The aim of the present study was to determine the association between hUCMSCs-derived exosomal miR-146a and LAMC2 in OC cells, and to examine the relevance of exosomal miR-146a/LAMC2 in TAX- and DTX-resistant OC. 


\section{Materials and methods}

Reagents, antibodies and lentiviral vectors. The following antibodies were used to identify hUCMSC surface markers: FITC-conjugated anti-CD90 (cat. no. 13801), anti-CD14 (cat. no. 29943), anti-CD73 (cat. no. 13160) and anti-CD105 (cat. no. 14606), and phycoerythrin (PE)-conjugated anti-CD34 (cat. no. 79253), anti-CD29 (cat. no. 34971), anti-CD44 (cat. no. 38200), anti-CD45-PC7 (cat. no. 28418) and anti-human leukocyte antigen DR-1 (HLA-DR; cat. no. 17634) (all Cell Signaling Technology, Inc.). The hUCMSC osteogenic differentiation kit OriCell ${ }^{\mathrm{TM}}$ (OriCell ${ }^{\mathrm{TM}}$; cat. no. HUXUC-90021) and adipogenic differentiation kit (cat. no. GUXMX-90031) were obtained from Cyagen Biosciences, Inc. ELISA kits for the detection of PI3K (cat. no. ab207485) and Akt (cat. no. ab176657) were purchased from Abcam. miR-146a inhibitor and inhibitor control were purchased from Shanghai GenePharma Co., Ltd. LAMC2 lentiviral overexpression vector (cat. no. RC222076L4) and empty vector control were purchased from OriGene Technologies, Inc. The specific sequences were: miR-146a inhibitor, 5'-CCCAUGGAAUUC AGUUCUCA-3'; and inhibitor control, 5'-CAGAGGUCA GGCCUUGCAAA-3'.

Bioinformatics analysis. Gene Expression Profiling Interactive Analysis (http://gepia.cancer-pku.cn/) was used to analyze the expression levels of LAMC2 in tumor tissues in The Cancer Genome Atlas-Ovarian Cancer (TCGA-OV) database (https://cancergenome.nih.gov) and normal ovarian tissues in the GTEX (https://www.gtexportal.org/) database. Significant differences were determined by Limma analysis (version 3.11; https://bioconductor.org/packages/limma/).

Cell culture and identification of hUCMSCs. hUCMSCs (SC2020011402) were purchased from FenghuiShengwu and cultured at $37^{\circ} \mathrm{C}$ with $5 \% \mathrm{CO}_{2}$ in DMEM (cat. no. 11965-084; Gibco; Thermo Fisher Scientific, Inc.) supplemented with 10\% FBS (cat. no. 10099141; Gibco; Thermo Fisher Scientific, Inc.), $100 \mathrm{~g} / \mathrm{l}$ streptomycin and $100 \mathrm{U} / \mathrm{ml}$ penicillin (Sangon Biotech Co., Ltd.). Subsequently, cells were blocked with 5\% bovine serum albumin (cat. no. E661003; Sangon Biotech Co., Ltd.) at $37^{\circ} \mathrm{C}$ for $40 \mathrm{~min}$ and incubated with a series of mouse anti-human FITC-conjugated anti-CD90, anti-CD14, anti-CD73, anti-CD105 and anti-CD29, and PE-conjugated anti-CD34, anti-CD44 and anti-HLA-DR antibodies (all dilutions, $1: 50$ ) at $37^{\circ} \mathrm{C}$ for $2 \mathrm{~h}$. A flow cytometer (BD FACSCanto II; BD Biosciences) was utilized for analysis using FlowJo v10.0 software (BD Biosciences). Following culturing with culture media from the hUCMSC osteogenic and adipogenic differentiation kits, hUCMSCs were stained with $0.1 \%$ alizarin red stain and $0.5 \%$ oil red $\mathrm{O}$ stain at $37^{\circ} \mathrm{C}$ for $2 \mathrm{~h}$.

Extraction and identification of exosomes. The extraction and identification of hUCMSCs-derived exosomes was performed as described in Data S1. The isolated exosomes were labeled using the PKH26 red fluorescent cell labelling kit (Sigma-Aldrich; Merck KGaA) according to the manufacturer's protocol. Subsequently, unlabeled or PKH26 red fluorescence-labeled exosomes were added to A2780 and SKOV3 cell lines and incubated at $37^{\circ} \mathrm{C}$ with $5 \% \mathrm{CO}_{2}$ for $24 \mathrm{~h}$. The cells were then fixed at room temperature with $4 \%$ paraformaldehyde for $30 \mathrm{~min}$ and stained with 4',6-diamidino-2-phenylindole at room temperature for $5 \mathrm{~min}$. The phagocytose of exosomes from SKOV3 and A2780 cell lines was observed using a confocal microscope (Zeiss AG).

Western blotting. The exosome marker proteins CD9 (1:1,000; cat. no. ab92726), CD81 (1:1,000; cat. no. ab79559), CD63 (1:1,000; cat. no. ab217345), heat shock protein 70 (HSP70; 1:1,000; cat. no. ab2787) and GAPDH (1:100; cat. no. ab8245) were detected by western blotting as previously described (16). All antibodies were from purchased from Abcam. Briefly, radioimmunoprecipitation assay lysis buffer containing phenylmethanesulfonyl fluoride (Sangon Biotech Co., Ltd.) was used to extract exosomes. Pierce ${ }^{\mathrm{TM}} 660 \mathrm{~nm}$ protein assay reagent (Thermo Fisher Scientific, Inc.) was applied to determine the protein concentration. The same amount of protein was isolated using $10 \%$ Gradi-Gel $^{\mathrm{TM}}$ II gradient gel electrophoresis (ElpisBiotech, Inc.) from exosomes $(20 \mu \mathrm{g})$ and cell lysate $(60 \mu \mathrm{g})$ and transferred to a polyvinylidene fluoride membrane (EMD Millipore). The membrane was subsequently blocked at room temperature for $1 \mathrm{~h}$ in tris buffer saline- $0.1 \%$ tween 20 containing 5\% bovine serum albumin and incubated overnight at $4{ }^{\circ} \mathrm{C}$ with the primary antibodies $(1: 2,000)$. The goat anti-mouse secondary antibody against IgG (cat. no. sc-2005; 1:5,000; Santa Cruz Biotechnology, Inc.) conjugated to horseradish peroxidase was added for a 1-h incubation at room temperature. Protein blots were visualized using an enhanced chemiluminescence kit (Santa Cruz Biotechnology, Inc.) and analyzed using Glyko BandScan 5.0 software (Agilent Technologies, Inc.).

Construction of OC cell lines resistant to chemotherapeutics. The establishment of OC resistant cell lines SKOV3/DTX and A2780/TAX is described in Data S1.

Cell treatment. Parental and resistant A2780 and SKOV3 cells in the logarithmic growth phase were selected for transfection using $100 \mathrm{ng}$ miR-146a inhibitor or corresponding inhibitor control with Lipofectamine 2000 (Invitrogen; Thermo Fisher Scientific, Inc.) according to the manufacturer's protocol. Reverse transcription-quantitative (RT-q)PCR was used to verify the success of transfection at $48 \mathrm{~h}$ post-transfection. Subsequently, cells were treated using $20 \mu \mathrm{g} / \mathrm{ml}$ hUCMSC-derived exosomes. Cells were collected after $24 \mathrm{~h}$ for subsequent experiments.

Cell survival assay. SKOV3 and A2780 cells were plated in 96-well plates at a density of $8 \times 10^{3}$ cells/well. After $4 \mathrm{~h}$, the cells were treated with either DTX (serial dilution, 0-5 $\mu \mathrm{M}$; Sigma-Aldrich; Merck KGaA) or TAX (serial dilution, 0-10 $\mu \mathrm{M}$; Sigma-Aldrich; Merck KGaA) for $72 \mathrm{~h}$ at $37^{\circ} \mathrm{C}$. A Cell Counting Kit-8 (CCK-8; Roche Diagnostics) assay was used to measure cell survival according to the manufacturer's protocol.

CCK-8 assay. Cells ( $5 \times 10^{3}$ cells/well) were treated with $10 \mu \mathrm{l}$ CCK-8 (Roche Diagnostics) for $2 \mathrm{~h}$ at $37^{\circ} \mathrm{C}$ followed by cell proliferation assays. The proliferation of cells was evaluated after $0,24,48$ or $72 \mathrm{~h}$ of incubation at $37^{\circ} \mathrm{C}$ according to the manufacturer's protocol. 
5-Ethynyl-2'-deoxyuridine (EdU) labelling. EdU analysis was performed according to the instructions of the Cell-Light ${ }^{\mathrm{TM}}$ EdU apollo643 in vitro kit (cat. no. C10310-1; Guangzhou RiboBio Co., Ltd.). After fixation with $4 \%$ paraformaldehyde (Sigma-Aldrich; Merck KGaA) for $30 \mathrm{~min}$ at $37^{\circ} \mathrm{C}$, the cells were treated with Apollo reaction mixture for $30 \mathrm{~min}$ at $37^{\circ} \mathrm{C}$ and stained with 4',6-diamidino-2-phenylindole at $37^{\circ} \mathrm{C}$ for $2 \mathrm{~h}$ for DNA staining. A2780 and SKOV3 cell proliferation was analyzed using randomly selected images obtained under a fluorescence microscope and was expressed as the ratio of $\mathrm{EdU}^{+}$cells to all cells.

Apoptosis analysis. Flow cytometry was used to detect apoptosis using a FITC-Annexin V Apoptosis Detection kit (BD Biosciences) according to the manufacturer's protocol. Apoptotic cells were loaded onto a BD FACSCanto II flow cytometer (BD Biosciences) and evaluated using FlowJo v10.0 software (BD Biosciences). In addition, SKOV3, SKOV3/DTX, A2780 and A2780/TAX cells $\left(1 \times 10^{6}\right.$ cells $\left./ \mathrm{ml}\right)$ were stained with $1 \mathrm{X}$ Hoechst 33258 staining solution at room temperature for 3-5 min. Apoptotic cells were observed using a Hoechst 33258 staining kit (Thermo Fisher Scientific, Inc.) after a 48-h exosome treatment at $37^{\circ} \mathrm{C}$.

Oligonucleotide microarray. Three groups of PBS-treated and MSC-derived exosome-treated SKOV3/DTX cells were collected and total RNA was extracted using TRIzol reagent (Thermo Fisher Scientific, Inc.). Subsequently, $0.5 \mu \mathrm{g}$ RNA was used for hybridization using the Human miRNA Expression Array V4.0 (Arraystar, Inc.). The extracted and isolated miRNAs were subsequently washed and scanned using the GeneChip ${ }^{\mathrm{TM}}$ Scanner3000 7G system (Thermo Fisher Scientific, Inc.).

$R T-q P C R$. Total RNA was extracted from cells using TRIzol (Invitrogen; Thermo Fisher Scientific, Inc.) and cDNA was synthesized using the Transcriptor First Strand cDNA Synthesis kit (Toyobo Life Science) at $42^{\circ} \mathrm{C}$ for $50 \mathrm{~min}$ and at $72^{\circ} \mathrm{C}$ for $10 \mathrm{~min}$. A total of $2 \mu \mathrm{g}$ cDNA was used as a template for qPCR to detect the target genes using SYBR Green Real-Time PCR master mix (Thermo Fisher Scientific, Inc.). The thermocycling conditions were: $95^{\circ} \mathrm{C}$ for $30 \mathrm{sec}$, followed by 40 (two-step) cycles of $95^{\circ} \mathrm{C}$ for $5 \mathrm{sec}$ and $60^{\circ} \mathrm{C}$ for $35 \mathrm{sec}$, and a final extension at $72^{\circ} \mathrm{C}$ for $15 \mathrm{~min}$. mRNA expression levels were measured using the $2^{-\Delta \Delta \mathrm{Cq}}$ method (17) after normalization to the expression levels of U6 or GAPDH. The primer sequences used were: miR-146a forward, 5'-TGAGAA CTGAATTCCATGGGT-3' and reverse, 5'-TATGGCACT GGTAGAATTCACT-3'; U6 forward, 5'-GACCTCTATGCC AACACAGT-3' and reverse, 5'-AGTACTTGCGCTCAGGA GGA-3'; LAMC2 forward, 5'-TACCAGAGCCAAGAACG CTG-3' and reverse, 5'-CGCAGTTGGCTGTTGATCTG-3'; and GAPDH forward, 5'-CCACTAGGCGCTCACTGTTCTC-3' and reverse, 5'-CATGGTGGTGAAGACGCCAG-3'.

Luciferase gene reporter assay. Initially, the targeting mRNAs of miR-146a were screened using StarBase (http://starbase.sysu.edu.cn/) and miRsearch (https://www. exiqon.com/ls/Pages/). The complementary sequence for miR-146a was synthesized and inserted into a psiCHECK2 vector (Promega Corporation) downstream of the Renilla reporter gene to generate psiCHECK2-miR-146a. The LAMC2 3' untranslated region (3'UTR) containing the putative miR-146a binding sites was cloned into the same vector to create psiCHECK2-LAMC2. The psiCHECK2 vectors contained a second reporter gene (firefly luciferase) designed for end point lysis assays. The reporter plasmid (100 ng) was transfected into cells using Lipofectamine LTX (Thermo Fisher Scientific, Inc.). Luciferase activity was measured after $48 \mathrm{~h}$ using the dual-luciferase reporter assay (Promega Corporation). Values were normalized to firefly luciferase activity.

ELISA. Specific ELISA kits were used to determine the protein expression levels of PI3K and Akt in cell lysates according to the manufacturer's protocol.

Statistical analysis. SPSS version 21.0 (IBM Corp.) software was used for statistical analysis. Data are presented as the mean \pm standard deviation. Each assay was repeated at least three times, and the comparisons between two groups were performed using an unpaired t-test. When comparing one factor among multiple groups, a one-way ANOVA was utilized, and when comparing two factors among multiple groups, two-way ANOVA was applied, both were followed by Tukey's post hoc test. $\mathrm{P}<0.05$ was considered to indicate a statistically significant difference.

\section{Results}

Isolation and characterization of hUCMSCs and extracted exosomes. Flow cytometry was used to detect the expression levels of surface markers of hUCMSCs. The presence of CD29, CD44, CD73, CD90 and CD105 was confirmed; while the cells were negative for CD14, CD34, HLA-DR and CD45 (Fig. 1A). The cell surface marker proteins expressed by the purified hUCMSCs met the current criteria for the definition of MSCs according to the Minimal Criteria for Defining Multipotent MSCs (18). In addition, the adipogenic and osteogenic differentiation abilities of hUCMSCs were assessed by Oil-red $\mathrm{O}$ staining and alizarin-red staining, respectively (Fig. 1B-D).

The exosomes exhibited an elliptical or cup-shaped morphology under an electron transmission microscope, with a particle size of $\sim 100 \mathrm{~nm}$ (Fig. 1E). Western blotting illustrated that the expression levels of CD9, CD63, CD81 and HSP70 were upregulated in exosomes compared with cell lysates, and only a small quantity of GAPDH was expressed (Fig. 1F). Furthermore, the protein concentration of the extracted exosomes was $332.81 \mu \mathrm{g} / \mathrm{ml}$. According to the definition of Exo by Minimal Information for Studies of Extracellular Vesicles 2018 (19), the extracted exosomes were considered to be extracellular vesicles. Subsequently, the exosomes were diluted to $20 \mu \mathrm{g} / \mathrm{ml}$ for subsequent use. After co-culturing SKOV3 and A2780 cells for $24 \mathrm{~h}$ with labeled exosomes, a fluorescence microscope was used to observe the cells and it was shown that the cells had a large quantity of red fluorescence, which was primarily distributed in the cytoplasm. The results revealed that the exosomes were internalized by SKOV3 and A2780 cells (Fig. 1G). 
A
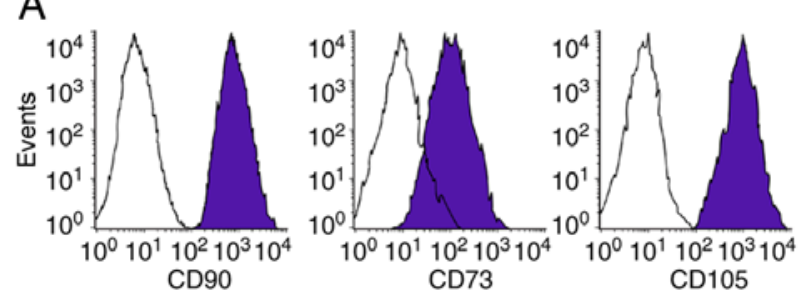

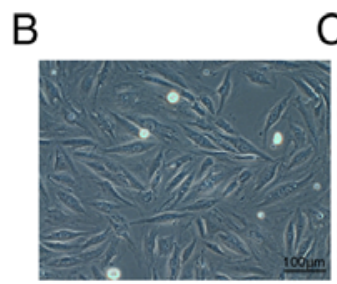

White light

E

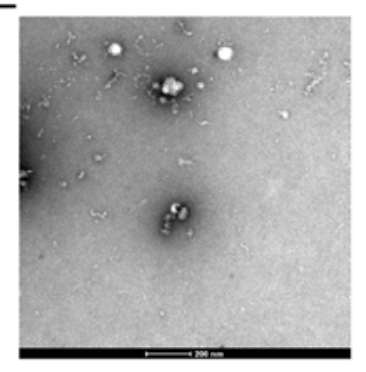

CD45
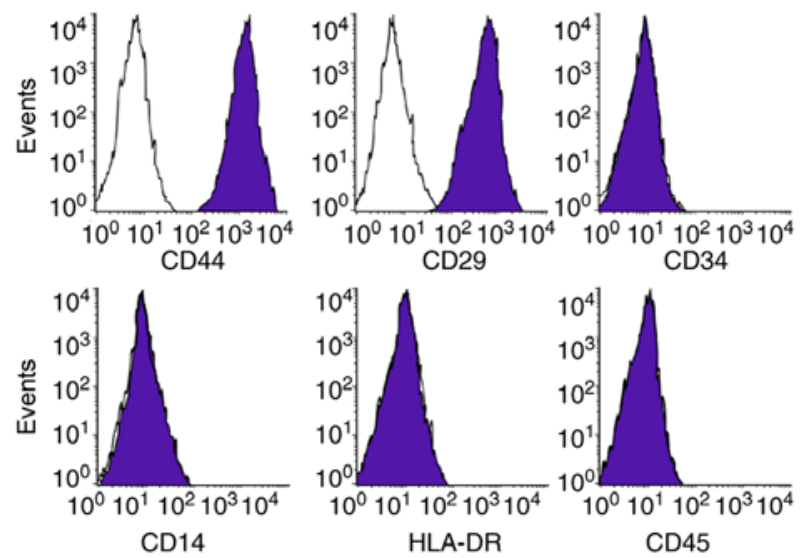

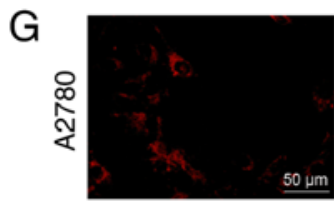

PKH-26

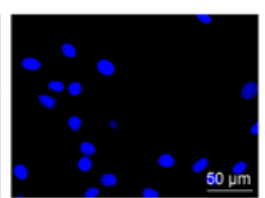

DAPI

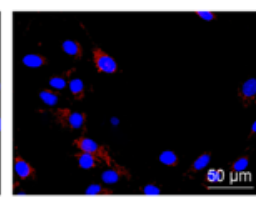

Merge

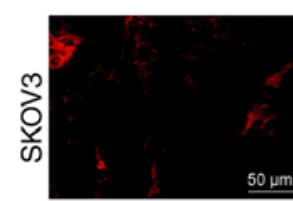

PKH-26

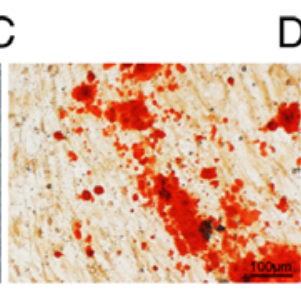

Alizarin red
D

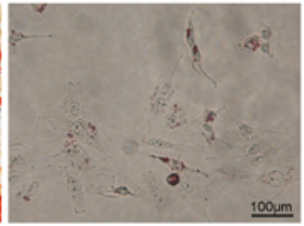

Oil O red

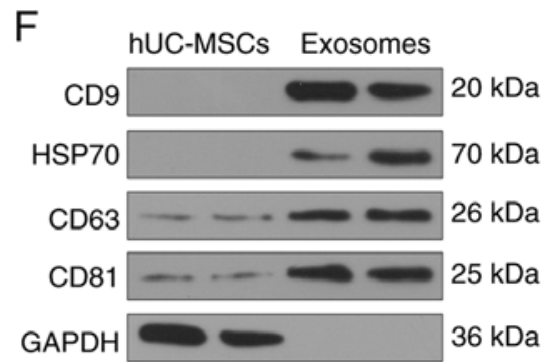

Figure 1. Identification of hUCMSCs and their derived exosomes. (A) Expression of MSCs surface markers, including CD29, CD44, CD73, CD90, CD105, CD14, CD34, CD45 and HLA-DR, analyzed by flow cytometry. (B) hUCMSC morphology at passage 3 observed under a light microscope. Scale bar, $100 \mu$ m. (C) Representative images of osteogenic differentiation of hUCMSCs using Alizarin Red staining. Scale bar, $100 \mu \mathrm{m}$. (D) Representative images of adipogenic differentiation of hUCMSCs using Oil red O staining. Scale bar, $100 \mu \mathrm{m}$. (E) Morphological analysis of hUCMSC by transmission electronic microscopy. Scale bar, $200 \mathrm{~nm}$. (F) Detection of exosomal marker expression in hUCMSC-released exosomes by western blotting. (G) Internalization of PKH26-labeled exosomes by SKOV3 and A2780 cells was observed under a fluorescence microscope. Scale bar, $50 \mu \mathrm{m}$. hUCMSCs, human umbilical cord mesenchymal stem cells.

hUCMSC-derived exosomes increase drug sensitivity in $O C$ cells. First, the successful induction of the SKOV3/DTX and A2780/TAX cell lines was assessed using a CCK-8 assay (P<0.05;Fig.2A).Subsequently, the extractedhUCMSC-derived exosomes were co-cultured with the parental SKOV3 or A2780 cells and the corresponding resistant cell lines at a dose of $20 \mu \mathrm{g} / \mathrm{ml}$ for $24 \mathrm{~h}$, and then cultured with $2 \mu \mathrm{M}$ DTX or $5 \mu \mathrm{M}$ TAX for $2 \mathrm{~h}$, followed by CCK-8, EdU staining and apoptosis detection experiments. The treatment of hUCMSC-derived exosomes reduced the resistance of OC parental cells to chemotherapy and the resistance of drug-resistant cells $(\mathrm{P}<0.05$; Fig. 2B-E). In general, hUCMSC-derived exosomes suppressed resistance to chemotherapy.

Expression of miR-146a is increased following treatment with exosomes. Microarray analysis was performed to analyze the differentially expressed miRNAs in SKOV3/DTX cells before and after exosome treatment. miR-146a expression was demonstrated to be increased following exosome treatment (Fig. 3A). Furthermore, RT-qPCR was used to detect the expression levels of miR-146a in parental SKOV3 and A2780 cells and the corresponding drug-resistant cell lines. A similar trend in miR-146a expression was observed in both cell lines relative to their parental SKOV3 and A2780 cells following treatment with exosomes (Fig. 3B). Therefore, to further clarify the role of miR-146a in OC cell chemoresistance, miR-146a inhibitor was transfected into exosome-treated parental SKOV3 and A2780 cells and the corresponding resistant cells (Fig. 3C).

Inhibition of miR-146a expression in OC cells reduces the effects of hUCMSC-derived exosomes. Exosome-treated parental SKOV3 and A2780 cells and the corresponding resistant cells were treated with $2 \mu \mathrm{M}$ DTX or $5 \mu \mathrm{M}$ TAX, respectively, and a CCK-8 assay, EdU staining and apoptosis analysis were performed. The results revealed that miR-146a inhibitor suppressed the sensitizing role of hUCMSC-derived exosomes in OC cells. Following knockdown of miR-146a in hUCMSCs, the drug resistance of SKOV3/DTX cells to DTX and the drug resistance of A2780/TAX to TAX were increased, the numbers of EdU positive cells were increased and the proportions of apoptotic cells were decreased in the parental and drug-resistant cells following treatment with $2 \mu \mathrm{M}$ DTX and $5 \mu \mathrm{M}$ TAX $(\mathrm{P}<0.05$; Fig. 4A-D). Therefore, transferring miR-146a may be one of the primary mechanisms by which hUCMSC-derived exosomes reduce chemoresistance.

miR-146a downregulates the PI3K signaling pathway by targeting LAMC2. To identify the targets of miR-146a that may have contributed to the increased chemosensitivity in $\mathrm{OC}$, the putative targets of miR-146a were predicted in silico 
A

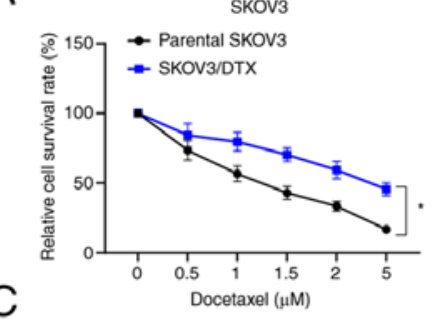

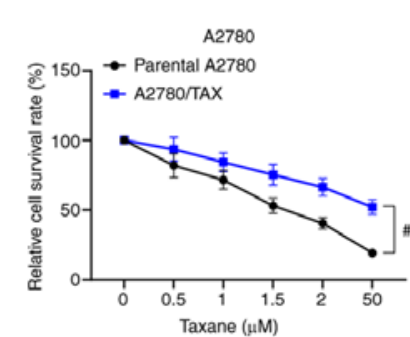

$2 \mu \mathrm{M}$ docetaxel

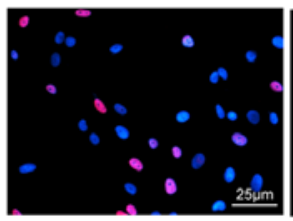

Parental SKOV $3+$ PBS

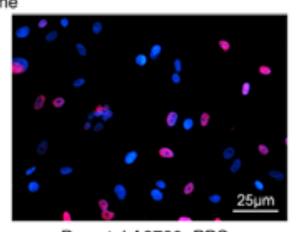

D Parental A2780+PBS

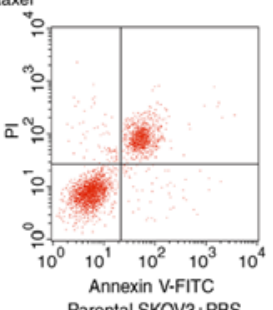

$5 \mu \mathrm{M}$ Taxane

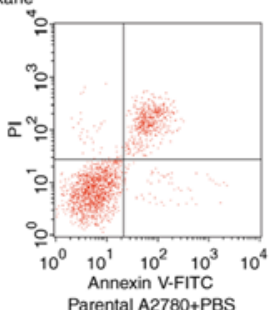

E

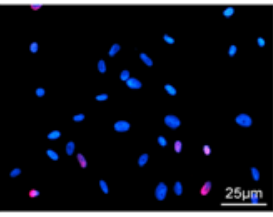

Parental SKOV3+Exosomes
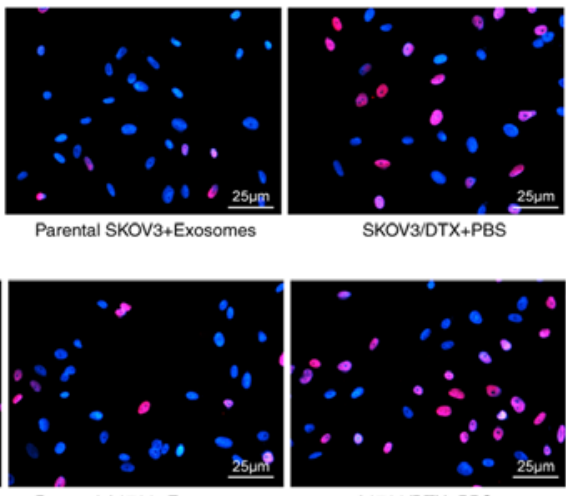

Parental A2780+Exosomes
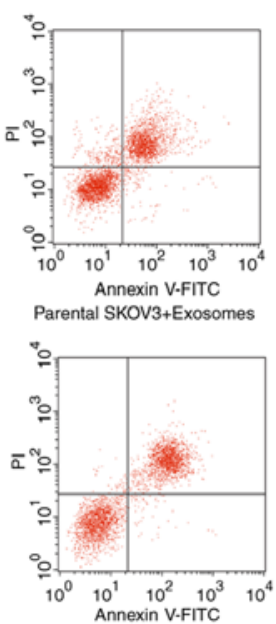

Parental A2780+Exosomes

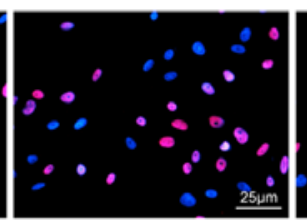

A2780/DTX+PBS
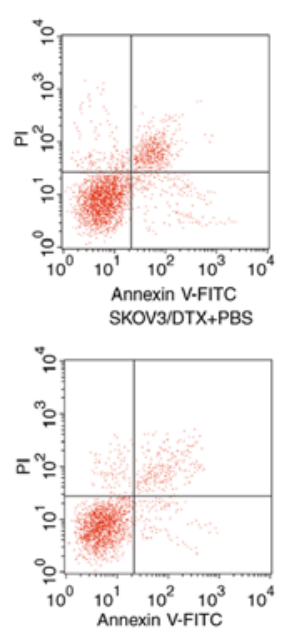

A2780/DTX+PBS
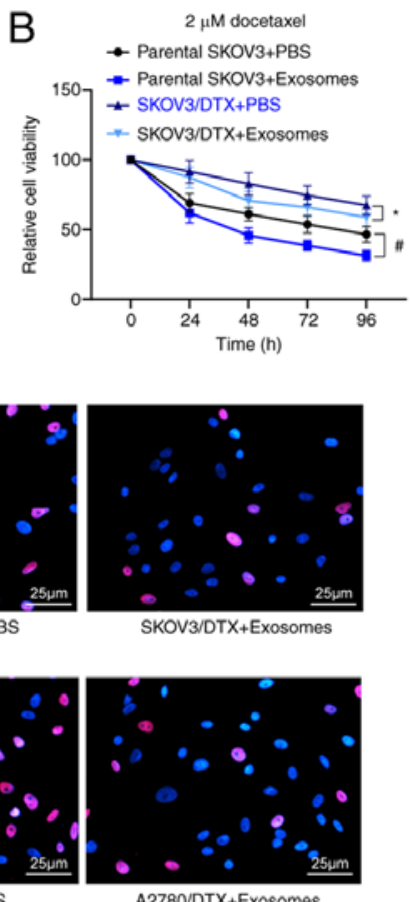

A2780/DTX+Exosomes

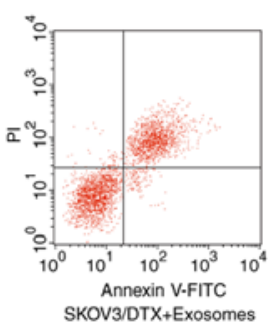

SKOV $3 /$ DTX+Exosomes

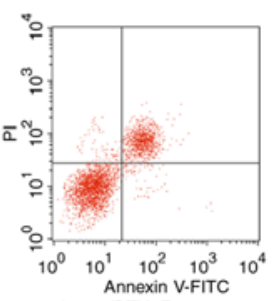

A2780/DTX+Exosomes

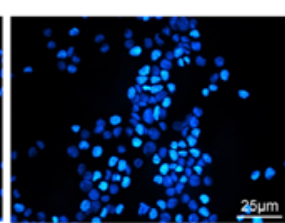

SKOV3/DTX+Exosomes

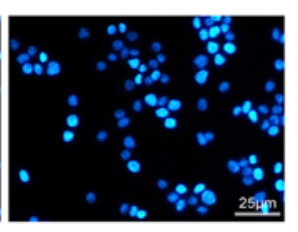

A2780/DTX+Exosomes
$5 \mu \mathrm{M}$ Taxane

- Parental A2780+PBS

- Parental A2780+Exosomes

$\leftarrow$ A2780/TAX+PBS
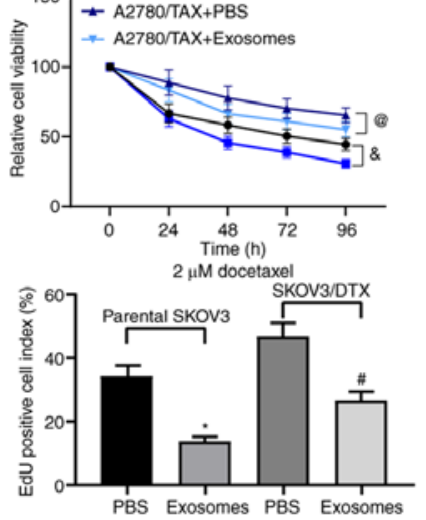

PBS Exosomes PBS Exosomes

$5 \mu \mathrm{M}$ Taxane

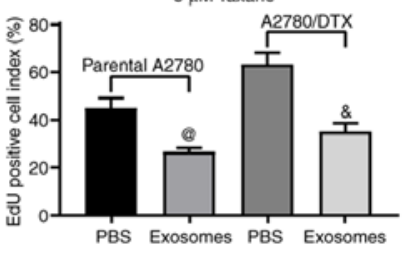

ஓ $2 \mu \mathrm{M}$ docetaxel
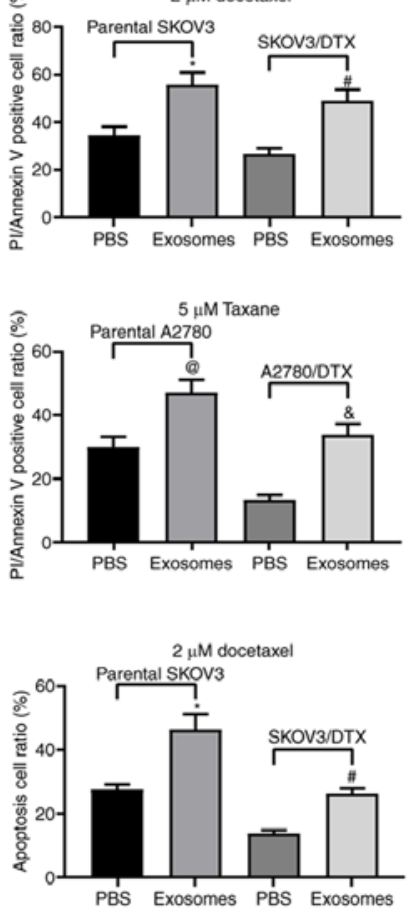
5 M Taxane

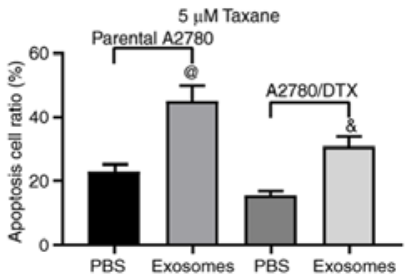

Figure 2. hUCMSC-derived exosomes inhibit OC cell drug resistance. SKOV3/DTX and A2780/TAX cells were induced by the exposure to gradient concentrations of drugs. (A) Cell survival rate examined by CCK- 8 cell survival assays. Subsequently, the parental or drug-resistant OC cells were treated with $20 \mu \mathrm{g} / \mathrm{ml}$ MSCs-derived exosomes or PBS as a negative control. Exosome-treated parental or drug-resistant OC cells were exposed to $2 \mu \mathrm{M}$ DTX or $5 \mu \mathrm{M}$ TAX for $2 \mathrm{~h}$, respectively. ${ }^{*} \mathrm{P}<0.05$ vs. parental SKOV3 cells, ${ }^{\text {}} \mathrm{P}<0.05$ vs. parental A2780 cells. (B) Cell viability evaluated by CCK-8 cell viability assays. (C) EdU staining for cell proliferation. Scale bar, $25 \mu \mathrm{m}$. (D) Flow cytometry analysis for cells labelled with PI/Annexin V. (E) Hoechst 33258 staining for cell apoptosis. Scale bar, $25 \mu \mathrm{m}$. Data are presented as the mean \pm SD. One-way (panels C, D and E) or two-way (panel A) ANOVA followed by Tukey's multiple comparisons test was used to determine statistical significance. Each assessment was performed in triplicate with three repetitions to ensure minimum deviation. In panels B, $\mathrm{C}$, D and E: ${ }^{*} \mathrm{P}<0.05$ vs. parental SKOV3 cells treated with PBS; ${ }^{~} \mathrm{P}<0.05$ vs. SKOV3/DTX cells treated with $\mathrm{PBS}$; ${ }^{\circ} \mathrm{P}<0.05$ vs. parental $\mathrm{A} 2780$ cells treated with PBS, ${ }^{\&}$ P<0.05 vs. A2780/TAX cells treated with PBS. CCK-8, Cell Counting Kit-8; DTX, docetaxel; hUCMSCs, human umbilical cord mesenchymal stem cells; OC, ovarian cancer; PI, propidium iodide; TAX, taxane. 


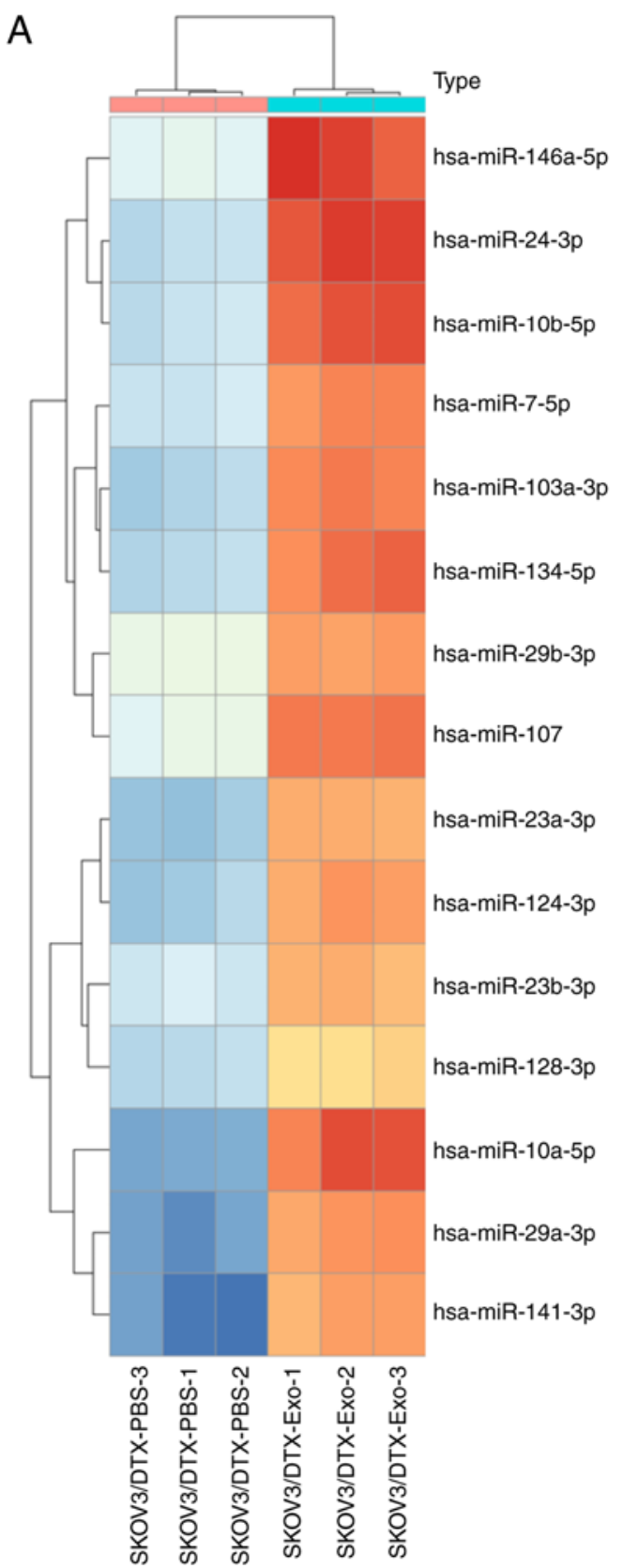

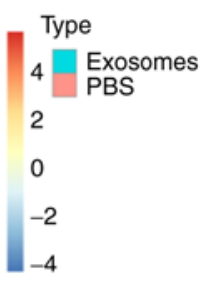
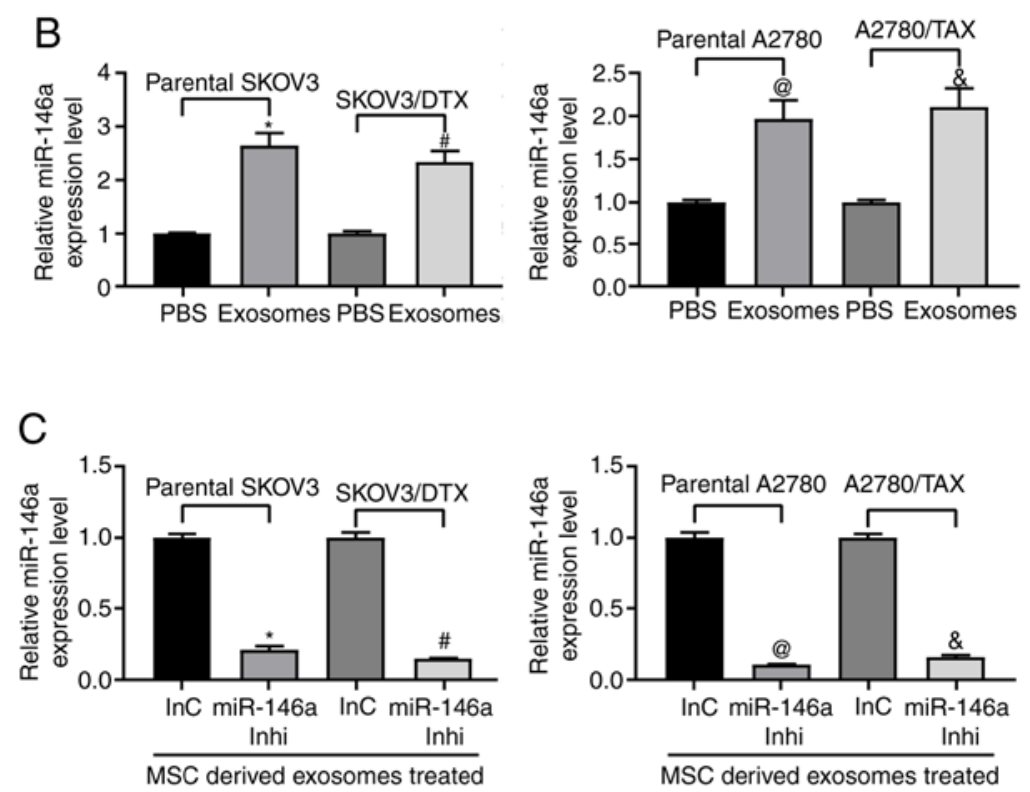

Figure 3. miR-146a is enriched in hUCMSC-derived exosomes. (A) Heatmap of 15 ectopic expressed miRNAs in hUCMSC-derived exosomes. (B) miR-146a expression in exosome-treated ovarian cancer cells. ${ }^{*} \mathrm{P}<0.05$ vs. parental SKOV3 cells treated with PBS; ${ }^{\text {P }}<0.05$ vs. SKOV3/DTX cells treated with PBS; ${ }^{\circledR} \mathrm{P}<0.05$ vs. parental A2780 cells treated with PBS; ${ }^{\&} \mathrm{P}<0.05$ vs. A2780/TAX cells treated with PBS. (C) miR-146a expression pattern in SKOV3 and A2780 cells treated with miR-146a inhibitor or inhibitor control and co-cultured with exosomes extracted from hUCMSCs. ${ }^{*}<0.05$ vs. exosomes co-cultured with parental SKOV 3 cells transfected with InC; ${ }^{\mathrm{P}}<0.05$ vs. exosomes co-cultured with SKOV3/DTX cells transfected with InC; ${ }^{\circledR} \mathrm{P}<0.05$ vs. exosomes co-cultured with parental A2780 cells transfected with InC; ${ }^{\&} \mathrm{P}<0.05$ vs. exosomes co-cultured with A2780/TAX cells transfected with InC. Data are presented as the mean \pm SD. One-way ANOVA and Tukey's multiple comparisons test were used to determine statistical significance. Each assessment was performed in triplicate with three repetitions to ensure minimum deviation. hUCMSCs, human umbilical cord mesenchymal stem cells; inhi, inhibitor; InC, inhibitor control; miR, microRNA.

using StarBase and miRSearch. Among the potential candidates, LAMC2 was selected for further analysis due to the complementary structures with miR-146a (Fig. 5A). To further identify whether miR-146a directly binds to the 3'UTR of LAMC2, chimeric constructs harboring LAMC2-wild type (LAMC2-WT) or LAMC2-mutant (LAMC2-MT) were constructed. As shown in Fig. 5B, overexpression of miR-146a inhibited the luciferase activity of the reporter gene in the WT construct but not the LAMC2-MT construct. Furthermore, gene expression profiling interactive analysis illustrated that LAMC2 expression was significantly increased in the TCGA-OV dataset compared with in normal ovarian tissues in the GTEX database (Fig. 5C). LAMC2 expression was upregulated in resistant $\mathrm{OC}$ cell lines, whereas, following the addition of exosomes, LAMC2 expression was significantly decreased. After further inhibition of miR-146a expression, LAMC2 expression was restored (Fig. 5D). ELISA was used to detect PI3K expression and Akt phosphorylation, and it was demonstrated that exosome treatment reduced PI3K expression and Akt phosphorylation, and miR-146a inhibitor 
A

B
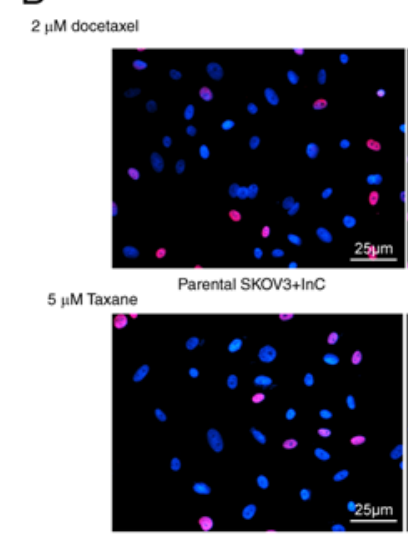

C

Parental A2780+InC
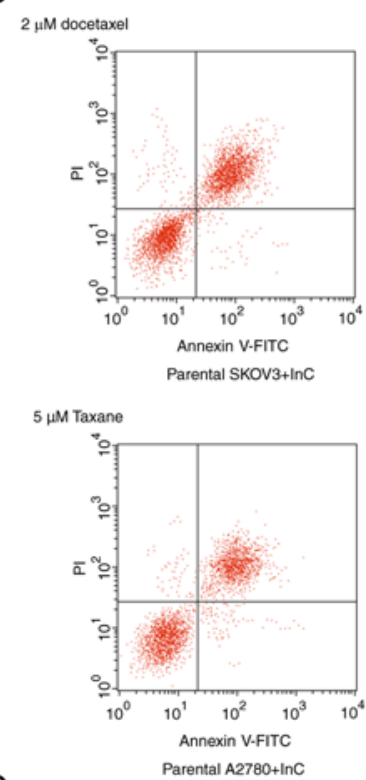

D

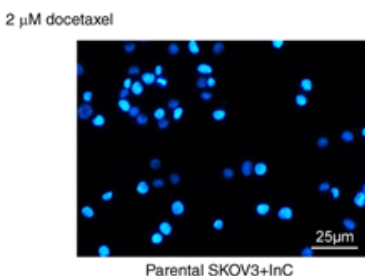

5 HM Taxane

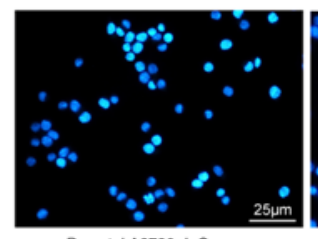

Parental A2780+InC
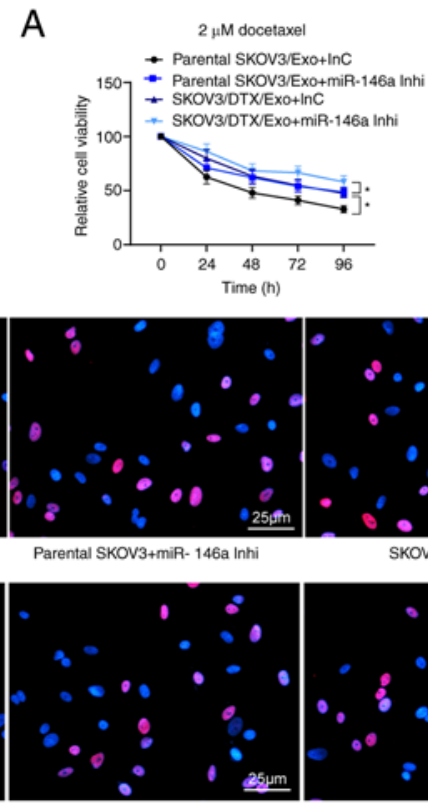

Parental A2780+miR-146a Inhi
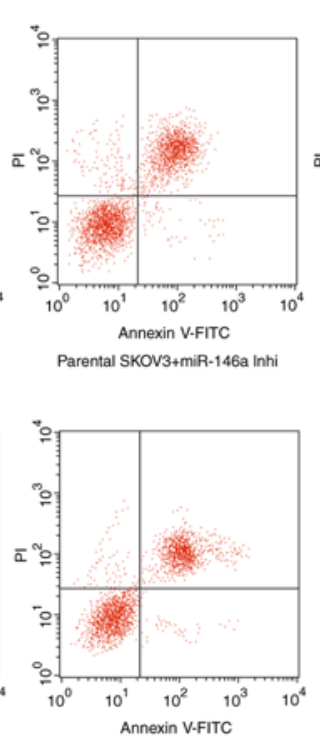

Parental A2780+miR-146a Inhi
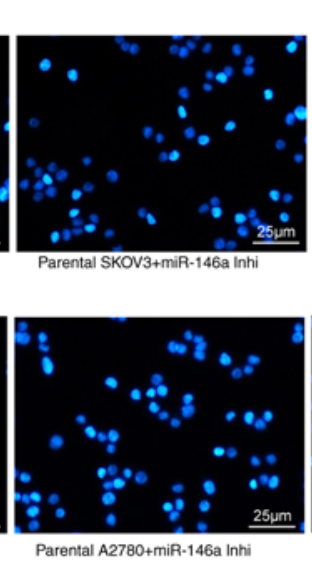
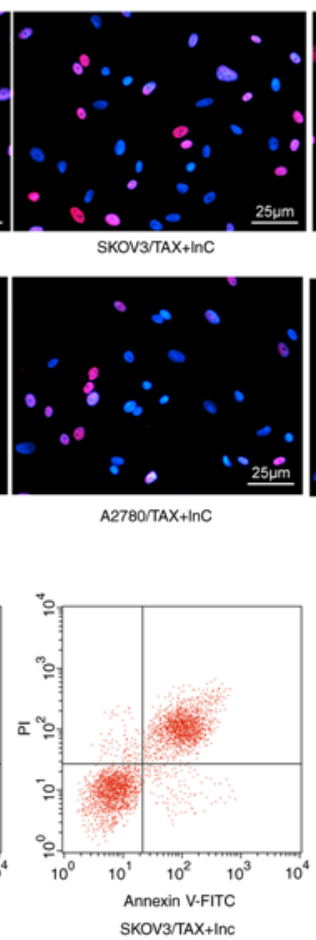

SKOV3/TAX+InC

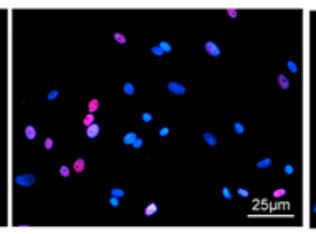

A2780/TAX+InC
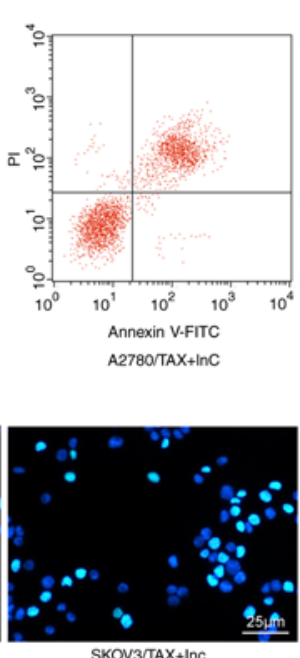

SKOV $3 /$ TAX+Inc

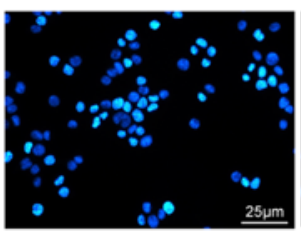

A2780/TAX+lnC
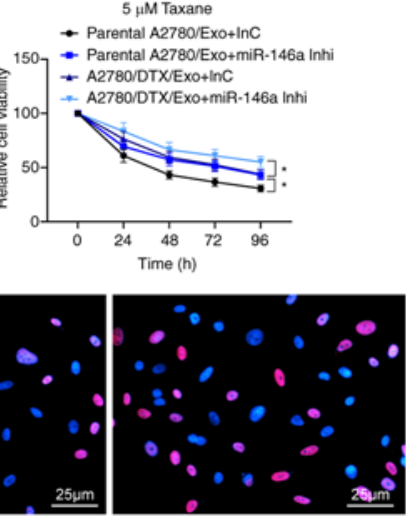

SKOV3/TAX+miR-146a Inhi

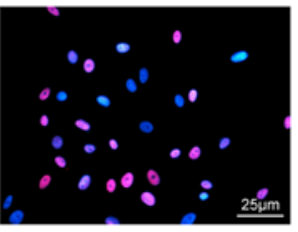

A2780/TAX+miR-146a Inhi
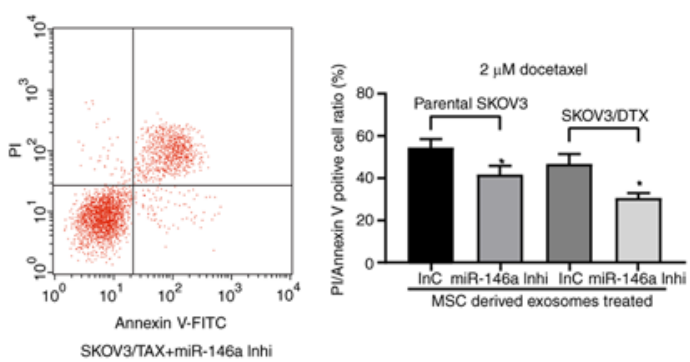
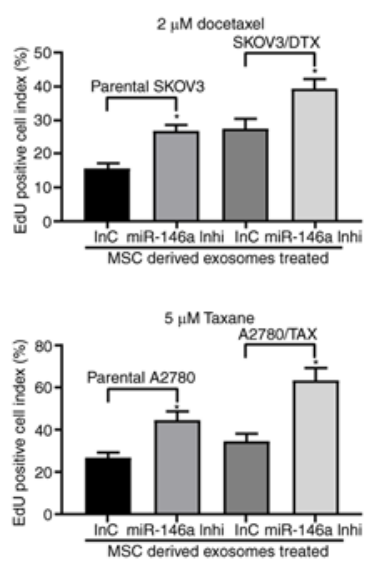

Figure 4. Exosomes from hUCMSCs promote OC cell chemosensitivity partly by transferring miR-146a. hUCMSC-derived exosome-treated parental or drug resistant OC cells were exposed to $2 \mu \mathrm{M}$ DTX or $5 \mu \mathrm{M}$ TAX for $2 \mathrm{~h}$, respectively. (A) Cell viability assessed by Cell Counting Kit- 8 cell viability assays. (B) EdU staining for cell proliferation. Scale bar, $25 \mu \mathrm{m}$. (C) Flow cytometry analysis for cells labelled with PI/Annexin V. (D) Hoechst 33258 staining for cell apoptosis. Scale bar, $25 \mu \mathrm{m}$. Data are presented as the mean \pm SD. One-way (panels C, D and E) or two-way (panel A) ANOVA followed by Tukey's post hoc test was used to determine statistical significance. Each assessment was performed in triplicate with three repetitions to ensure minimum deviation. ${ }^{*} \mathrm{P}<0.05$ vs. parental cells co-cultured with exosomes derived from InC-transfected MSCs. DTX, docetaxel; hUCMSCs, human umbilical cord mesenchymal stem cells; inhi, inhibitor; InC, inhibitor control; miR, microRNA; OC, ovarian cancer; PI, propidium iodide; TAX, taxane. 
A Alignment

Target: $\quad 5^{\prime}$ aaCCCAGGGUGUGACAUGUUCUCc 3 '

| || || : | || ||||||

miRNA : $\quad$ 3' uUGGGUACCUUA - - AGU - CAAGAGu 5'

B

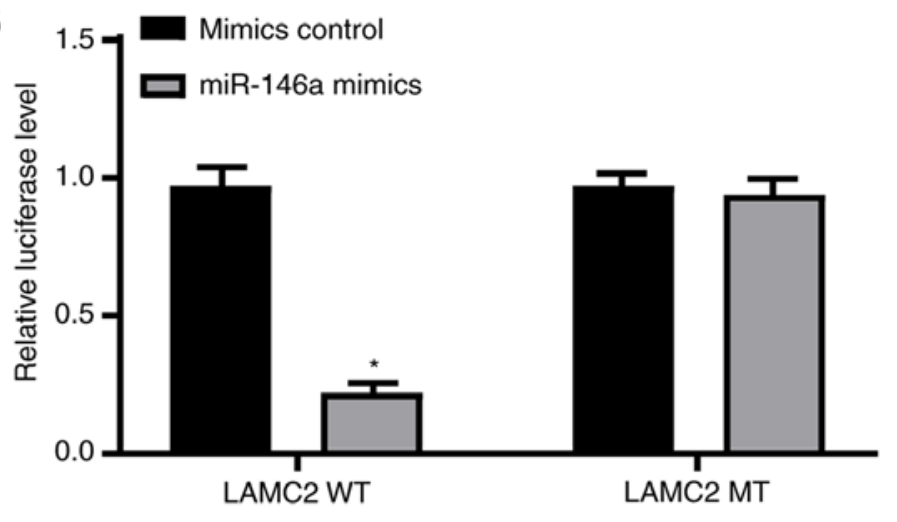

C

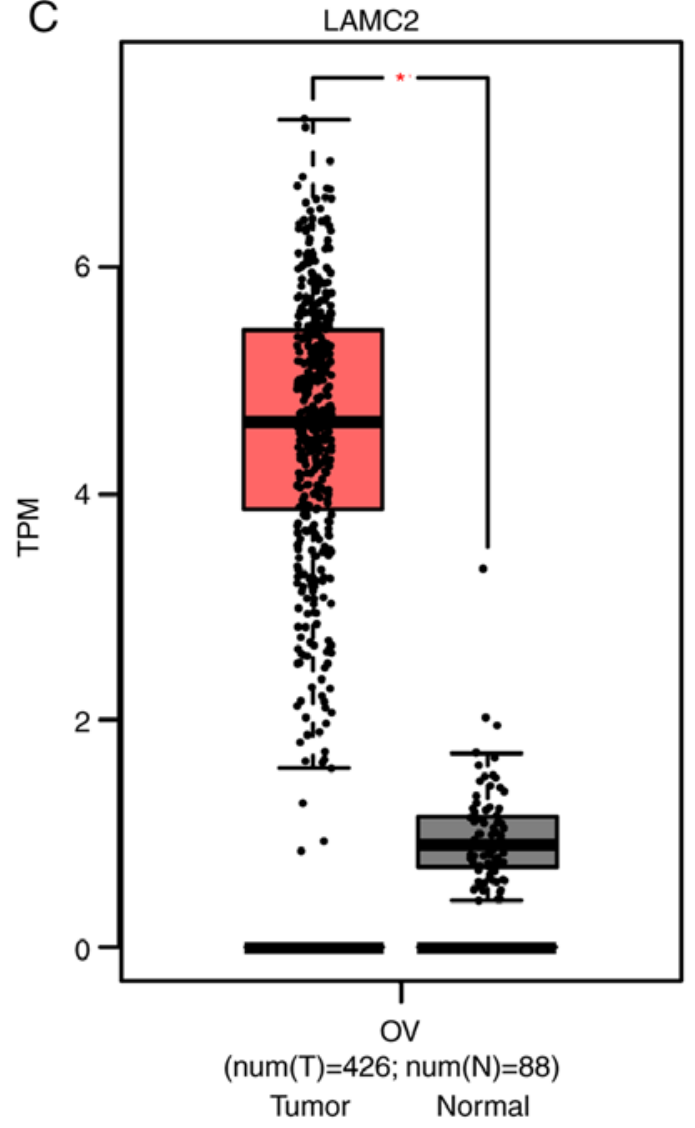

D
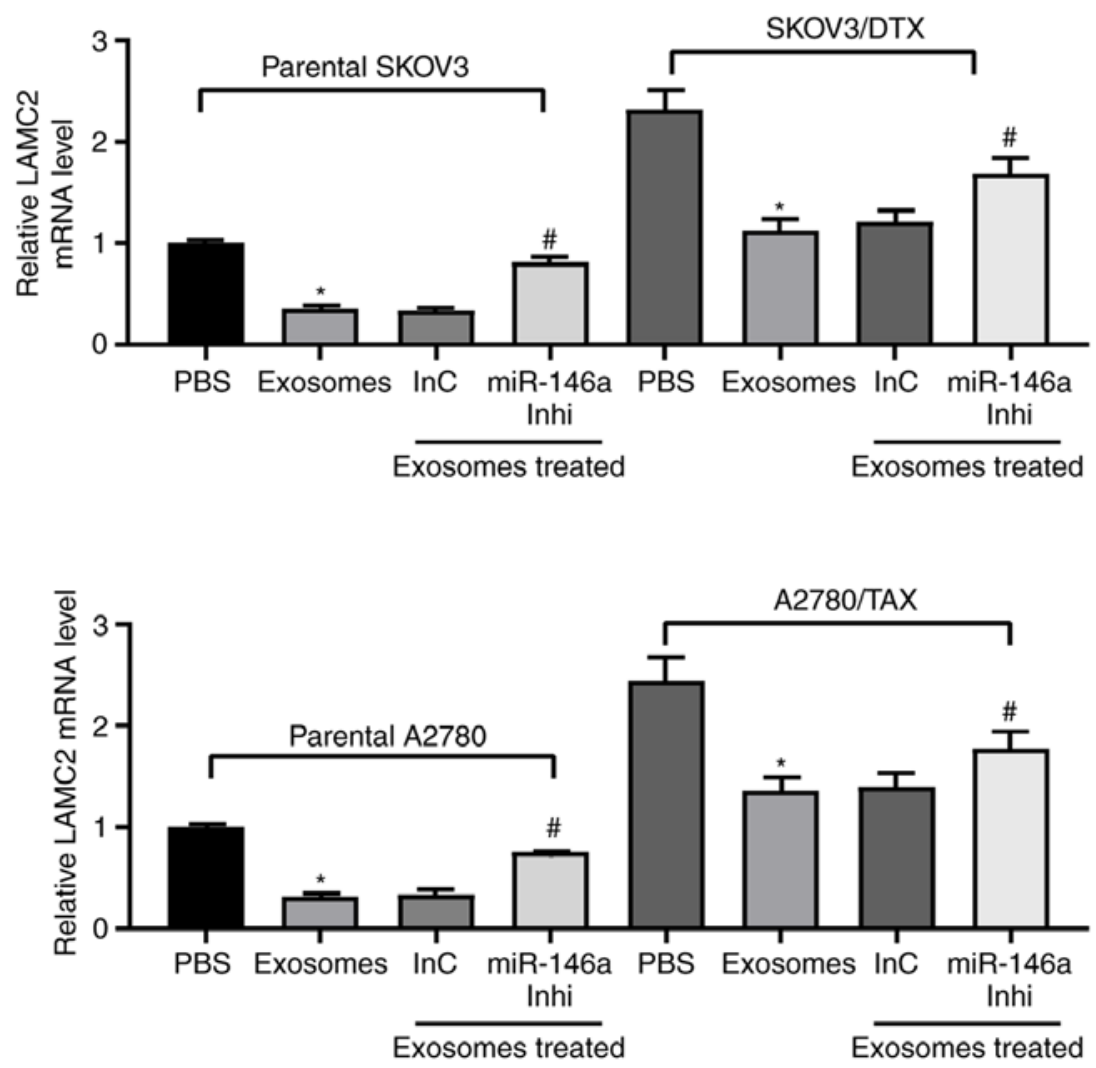

Figure 5. miR-146a blocks the PI3K signaling pathway by inhibiting LAMC2. (A) Possible miR-146 binding sites in the LAMC2 3' untranslated region. (B) Impact of miR-146a on the luciferase activity of LAMC2-WT and LAMC2-MT evaluated by dual luciferase assays. ${ }^{*} \mathrm{P}<0.05$ vs. LAMC2 MT. (C) LAMC2 expression in The Cancer Genome Atlas-ovarian cancer dataset ( $\mathrm{n}=426$ for tumor samples and $\mathrm{n}=88$ for normal tissues) predicted by gene expression profiling interactive analysis. "P<0.05. (D) LAMC2 expression in SKOV3 and A2780 cells determined by RT-qPCR. " $\mathrm{P}<0.05$ vs. cells treated with $\mathrm{PBS}$; ${ }^{\#} \mathrm{P}<0.05$ vs. cells co-cultured with exosomes extracted from InC-treated MSCs. 

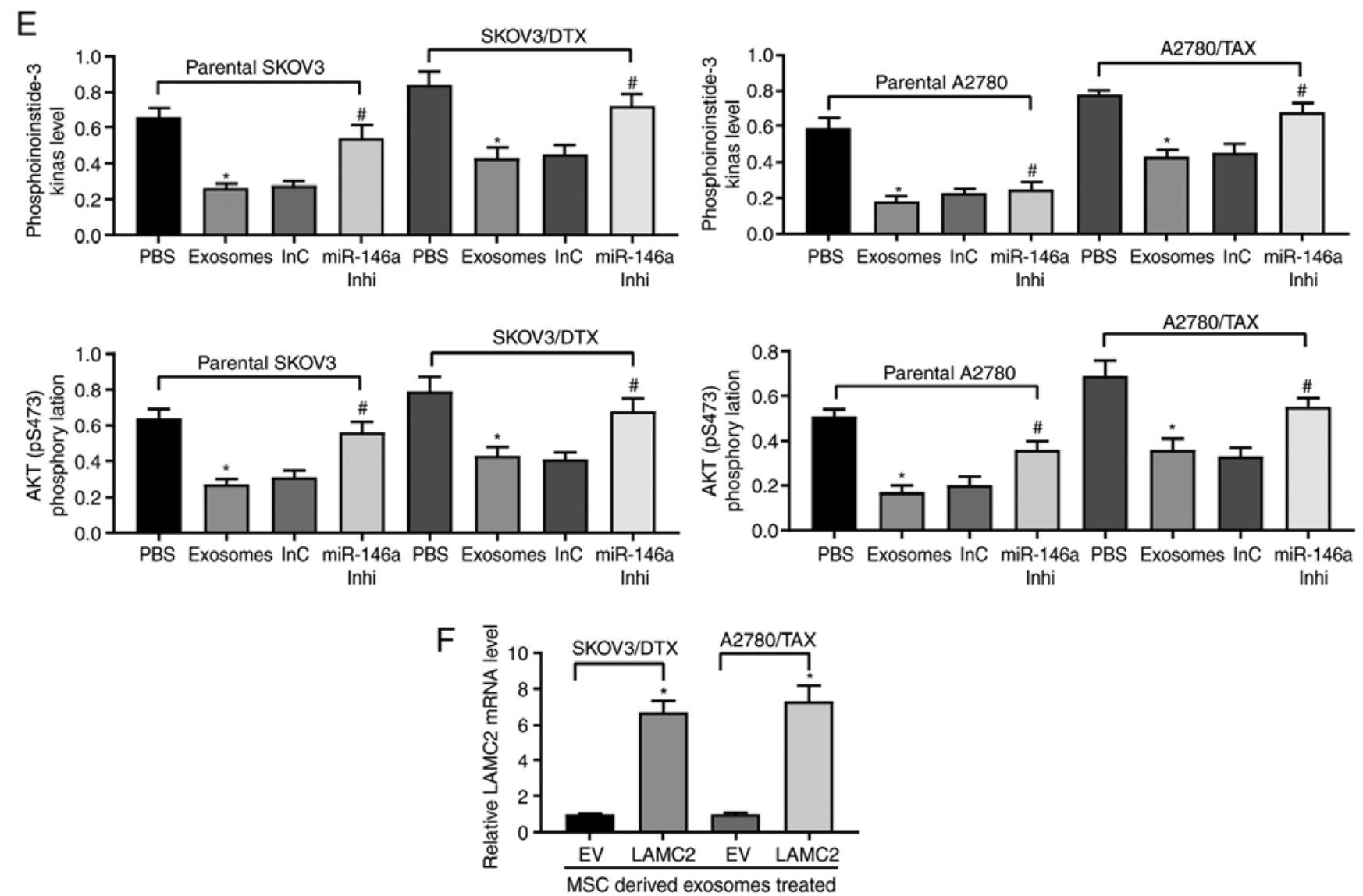

Figure 5. Continued. (E) Protein expression levels of PI3K measured by ELISA. * $\mathrm{P}<0.05$ vs. cells treated with PBS; ${ }^{*} \mathrm{P}<0.05$ vs. cells co-cultured with exosomes extracted from InC-treated MSCs. (F) LAMC2 expression in SKOV3/DTX and A2780/TAX cells determined by RT-qPCR. Data are presented as the mean $\pm \mathrm{SD}$. ${ }^{*} \mathrm{P}<0.05$ vs. cells transfected with EV. One-way (panels D, E and F) or two-way (panel B) ANOVA and Tukey's multiple comparisons test were used to determine statistical significance. Each assessment was performed in triplicate with three repetitions to ensure minimum deviation. DTX, docetaxel; InC, inhibitor control; inhi, inhibitor; LAMC2, laminin $\gamma 2$; miR, microRNA; MSCs, mesenchymal stem cells; MT, mutant; RT-qPCR, reverse transcription-quantitative PCR; TAX, taxane; WT, wild type.

treatment increased these (Fig. 5E). To determine the role of LAMC2 in OC cell resistance mediated by hUCMSC-released exosomes, LAMC2 was overexpressed in SKOV3/DTX and A2780/TAX cells. Transfection efficiency was determined by RT-qPCR (Fig. 5F).

Overexpression of LAMC2 reduces drug sensitivity in $O C$ cells. LAMC2 lentivirus-packed overexpression vector was added to exosome-treated parental SKOV3 and A2780 cells, and RT-qPCR was used to verify successful infection (Fig. 6A). Subsequently, the drug sensitivity of the cells to DTX and TAX was observed. Following overexpression of LAMC2, the drug resistance of OC cells treated with hUCMSC-derived exosomes was reduced, which was demonstrated by increased cell viability after DTX or TAX treatment (Fig. 6B) and increased numbers of EdU positive cells (Fig. 6C), but decreased numbers of apoptotic cells (Fig. 6D and E). Overall, these results revealed that hUCMSC-released exosomes reduced LAMC2 expression by transferring miR-146a, which is involved in the modulation of OC cell chemosensitivity.

\section{Discussion}

Exosomes, endogenous nanoparticles with a diameter of 40-100 $\mathrm{nm}$, are produced by most cell types and released into the extracellular space. Exosomes have been demonstrated to deliver mRNAs, miRNAs and other non-coding RNAs to neighboring cells and/or distant cells when in circulation (20). The use of MSC-secreted exosomes as biological vehicles to carry tumor suppressor miRNAs or chemotherapeutic agents is a potential approach for cancer treatment (21). In the present study, hUCMSCs effectively transferred miR-146a into secreted exosomes, which increased the sensitivity to the chemotherapeutic agents DTX and TAX by reducing LAMC2 expression via the PI3K/Akt signaling pathway in OC cells. The primary results suggested a feasible therapeutic regimen for use of hUCMSCs-derived exosomes as a potential vehicle for delivery of miR-146a to OC cells.

The present study demonstrated that hUCMSC-derived exosomes, successfully engineered to package miR-146a into the exosomes, has the potential to deliver miR-146 to OC cells in vitro. Exosomal miR-146a effectively reduced the chemoresistance of OC cells to DTX and TAX. In agreement with the results of the present study, Asare-Werehene et al (22) have demonstrated that exosome-transported plasma gelsolin results in increased chemoresistance in OC cells in an autocrine manner, and confers cisplatin resistance to chemosensitive OC cells (22). Furthermore, cancer-associated fibroblasts-secreted exosomes transferred miR-98-5p to potentiate $\mathrm{OC}$ cell resistance to cisplatin, which was associated with 

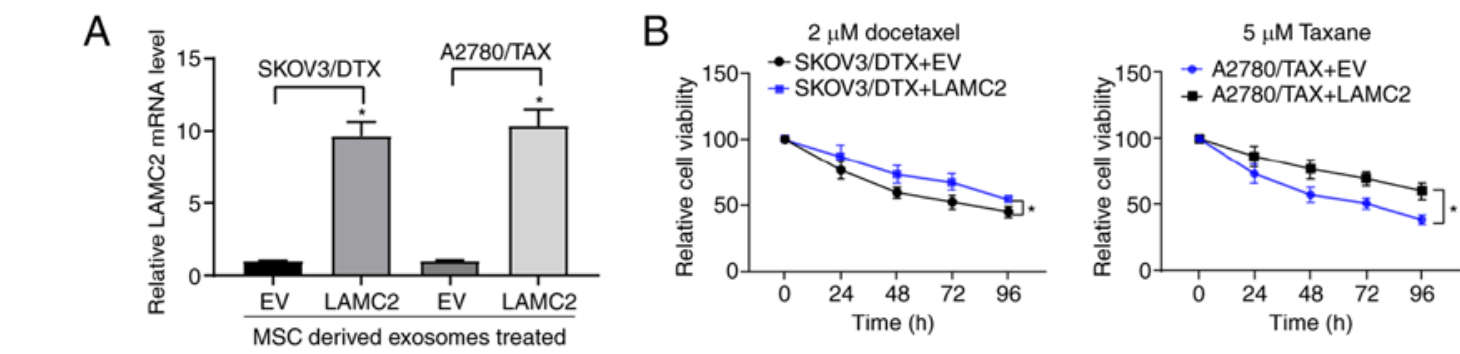

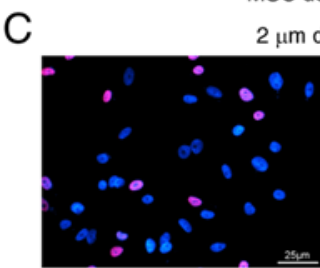

SKOV3/DTX+EV
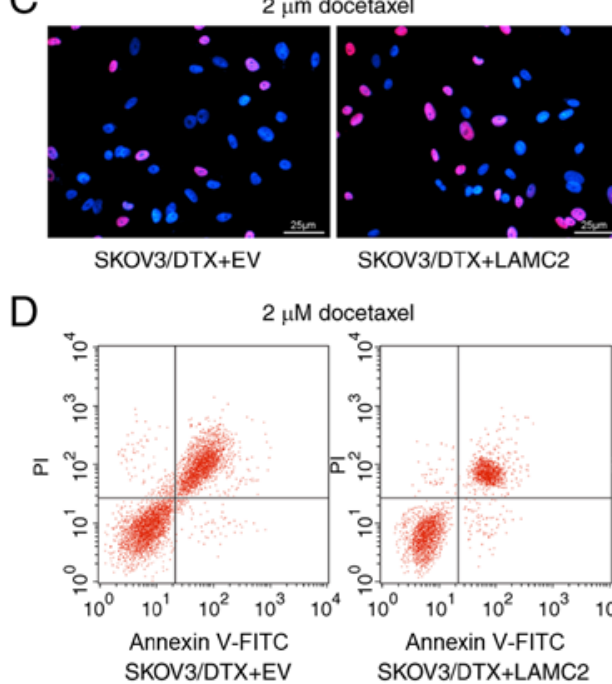

SKOV3/DTX+LAMC2

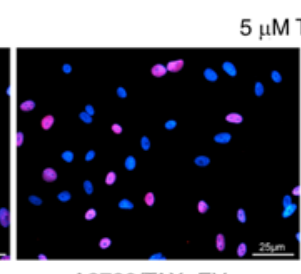

$\mathrm{A} 2780 / \mathrm{TAX}+\mathrm{EV}$

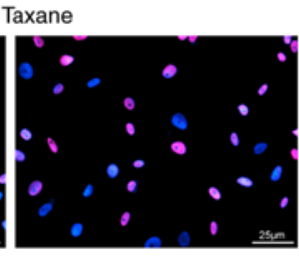

A2780/TAX+LAMC2
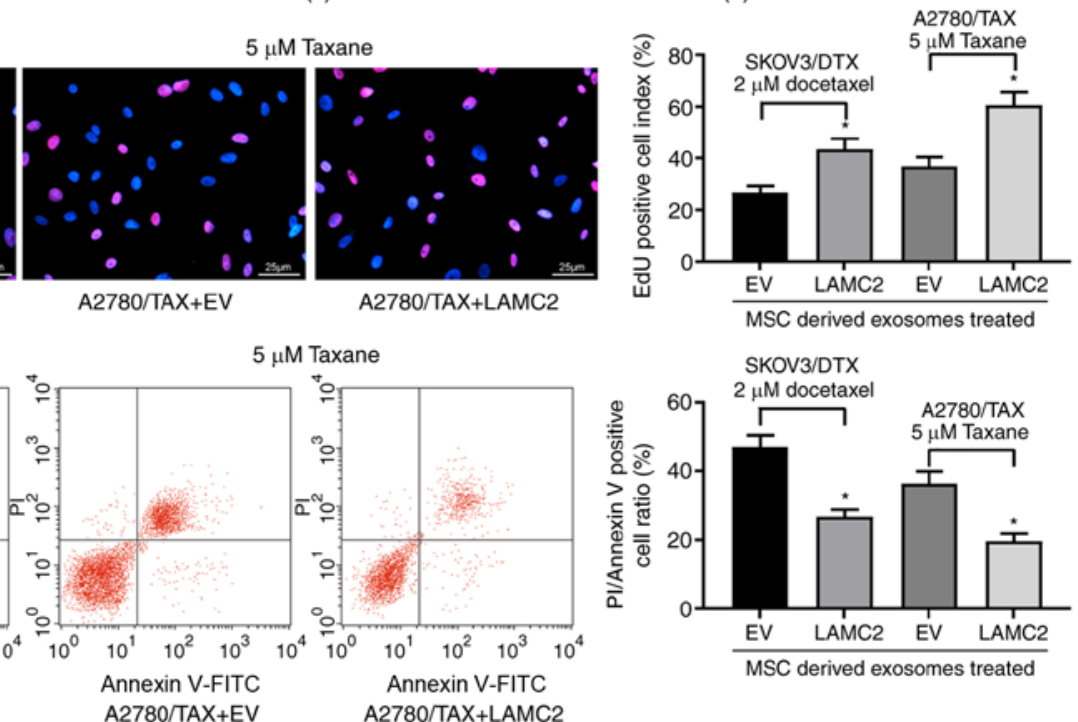

E

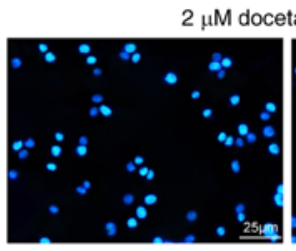

SKOV3/DTX+EV

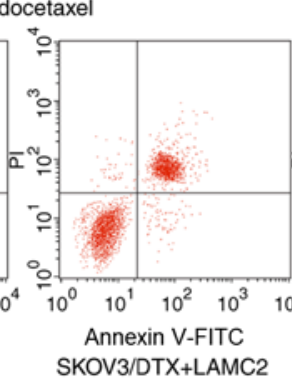

A2780/TAX+EV

A2780/TAX+LAMC2

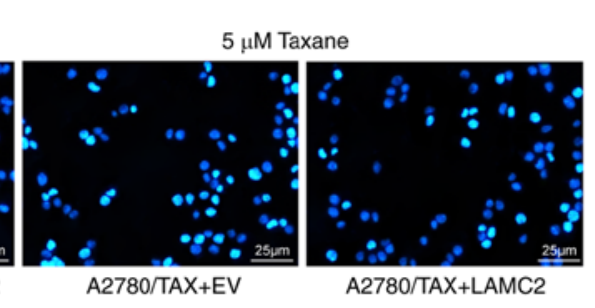

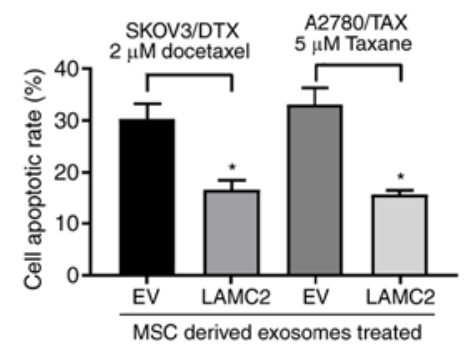

Figure 6. LAMC2 is involved in miR-146a-mediated OC cell sensitivity. hUCMSCs-derived exosome-treated parental or drug resistant OC cells were exposed to $2 \mu \mathrm{M}$ DTX or $5 \mu \mathrm{M}$ TAX for $2 \mathrm{~h}$, respectively. (A) LAMC2 mRNA expression measured by reverse transcription-quantitative PCR. (B) Cell viability examined by Cell Counting Kit-8 cell viability assays. (C) EdU staining of OC cells. Scale bar, $25 \mu \mathrm{m}$. (D) Flow cytometry analysis for cells labelled with propidium iodide/Annexin V. Scale bar, $25 \mu \mathrm{m}$. (E) Hoechst 33258 staining for cell apoptosis. Data are presented as the mean \pm SD. One-way (panels A, C, D and E) or two-way (panel B) ANOVA followed by Tukey's multiple comparisons test was used. ${ }^{*} \mathrm{P}<0.05$ vs. cells transfected with EV. Each assessment was performed in triplicate with three repetitions to ensure minimum deviation. DTX, docetaxel; EV, empty vector; hUCMSCs, human umbilical cord mesenchymal stem cells; LAMC2, laminin $\gamma 2$; miR, microRNA; OC, ovarian cancer; TAX, taxane.

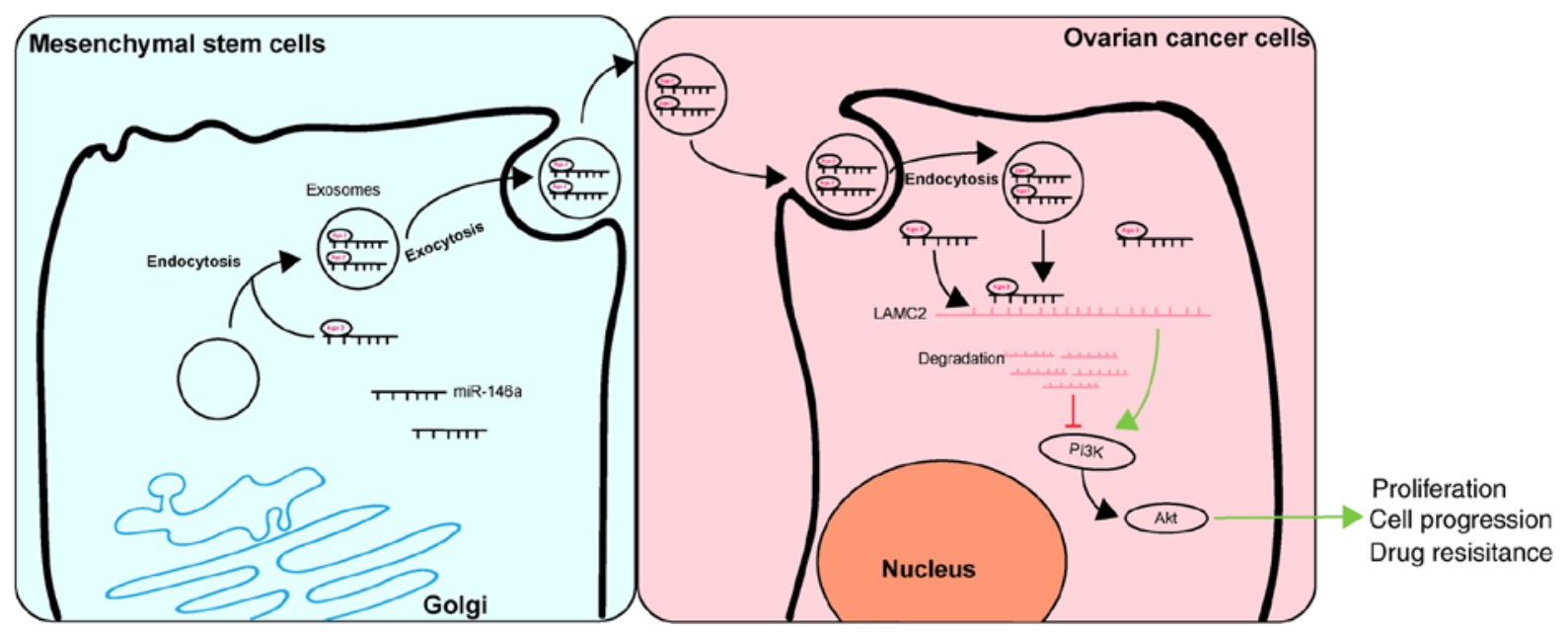

Figure 7. Regulatory mechanism of hUCMSC-derived exosomal miR-146a involved in OC cell proliferation and drug resistance. hUCMSC-derived exosomal miR-146a impeded the proliferation and drug resistance of OC cells by targeting LAMC2 to regulate the PI3K/Akt signaling pathway. hUCMSCs, human umbilical cord mesenchymal stem cells; miR, microRNA; OC, ovarian cancer. 
upregulated expression of the anticancer drug target CDKN1A in cisplatin-sensitive OC cells (23). Therefore, it was hypothesized that the exosomal content conferred chemoresistance in OC cells. Macrophage-secreted exosomes containing elevated levels of miR-146a-5p have been delivered to endothelial cells, and resulted in reduced migration of monocytes by targeting junctional adhesion molecule C (24). In vivo, circulating endothelial cell-derived extracellular vesicles from tumor-bearing mice have been demonstrated to contain upregulated levels of miR-146a compared with the control mice (25). Additionally, overexpression of miR-146a results in increased cell proliferation, and increased apoptosis and sensitivity to DTX in epithelial OC cells (26). Furthermore, hUCMSC-delivered exogenous miR-145-5p reduces pancreatic ductal adenocarcinoma cell proliferation and invasion, and increases apoptosis and cell cycle arrest (27). In vitro and in vivo assays have confirmed the tumor suppressor role of hUCMSCs-released exosomes carrying miR-148b-3p in breast cancer (28). The present study revealed that hUCMSC-delivered exosomes with increased expression levels of miR-146a sensitized the OC cells to DTX and TAX by targeting LAMC2.

LAMC2, a component of Ln-332, is a potent tumor biomarker which is upregulated in urothelial carcinoma (29), hepatocellular carcinoma (30) and pancreatic adenocarcinoma (31). In addition, Ln-332 increases chemoresistance and quiescence of cancer stem cells in hepatocellular carcinoma (32). The bioinformatics analysis performed in the present study suggested that LAMC2 expression was increased in TCGA-OV, and this upregulation was reversed by hUCMSCs-delivered exosomes. In addition, miR-146a inhibitor reduced the expression levels of LAMC2 in SKOV3 and A2780 parental and resistant cells. Furthermore, upregulation of LAMC2 significantly reduced the inhibitory role of hUCMSCs-delivered exosomes in OC cell chemoresistance, as suggested by the reduced apoptosis and increased proliferation, further demonstrating that LAMC2 was involved in the mediation of exosomal miR-146a in OC cell chemoresistance. Similarly, LAMC2 expression is upregulated and facilitates metastasis in esophageal squamous cell carcinoma, whereas LAMC2 restoration partially reduces the decrease in cell migration and invasion induced by cancer susceptibility 9 knockdown (33). The ELISA experiments performed in the present study revealed that exosome treatment reduced the activity of the PI3K/Akt signaling pathway in both resistant and sensitive OC cells, as demonstrated by the decreased PI3K expression and Akt phosphorylation levels; whereas miR-146a inhibitor reversed these trends. PI3K is the control hub of growth signal transmission, and activated PI3K is a hallmark of several types of cancer, including OC (34). Consistent with the results of the present study, PI3K gene expression is significantly increased in cervical cancer tissues and the derived exosomes relative to the corresponding adjacent tissues (35). Furthermore, PI3K has been reported to be a putative target of miR-146a in chondrocytes, whereas PI3K expression at both the protein and mRNA levels is decreased following transfection with a miR-146a mimic (36).

In conclusion, the results of the present study demonstrated that exosomal miR-146a released by hUCMSCs contributed to hUCMSC-derived exosome-mediated chemosensitivity, and this effect may be mediated by interactions between miR-146a and LAMC2 via the PI3K/Akt signaling pathway (Fig. 7).
These results may improve the understanding of the intercellular communications between cancer cells and MSCs through an MSC-exosome-miRNA axis. The present study suggests that management of hUCMSCs and their released exosomes may be a therapeutic strategy with potential clinical applications.

\section{Acknowledgements}

Not applicable.

\section{Funding}

No funding was received.

\section{Availability of data and materials}

All data generated or analyzed during this study are included in this published article.

\section{Authors' contributions}

LQ conceived and designed the present study. JW contributed to the design and definition of intellectual content of this study. MC, FC and WT contributed to the experimental studies, data acquisition and statistical analysis. LQ and WT contributed to the manuscript preparation. All authors read and approved the final manuscript and guarantee the integrity of the study.

\section{Ethics approval and consent to participate}

Not applicable.

\section{Patient consent for publication}

Not applicable.

\section{Competing interests}

The authors declare that they have no competing interests.

\section{References}

1. Tawde SA, Chablani L, Akalkotkar A and D'Souza MJ: Evaluation of microparticulate ovarian cancer vaccine via transdermal route of delivery. J Control Release 235: 147-154, 2016.

2. Zahedi P, De Souza R, Huynh L, Piquette-Miller M and Allen C: Combination drug delivery strategy for the treatment of multidrug resistant ovarian cancer. Mol Pharm 8: 260-269, 2011.

3. Lohse I, Azzam DJ, Al-Ali H, Volmar CH, Brothers SP, Ince TA and Wahlestedt C: Ovarian cancer treatment stratification using ex vivo drug sensitivity testing. Anticancer Res 39: 4023-4030, 2019.

4. Secord AA: Ovarian cancer: Time to move beyond one size fits all. Lancet Oncol 20: 754-755, 2019.

5. Borley $\mathbf{J}$ and Brown R: Epigenetic mechanisms and therapeutic targets of chemotherapy resistance in epithelial ovarian cancer. Ann Med 47: 359-369, 2015.

6. Ridge SM, Sullivan FJ and Glynn SA: Mesenchymal stem cells: Key players in cancer progression. Mol Cancer 16: 31, 2017.

7. Li P, Gong Z, Shultz LD and Ren G: Mesenchymal stem cells: From regeneration to cancer. Pharmacol Ther 200: 42-54, 2019.

8. Ding DC, Shyu WC, Lin SZ, Liu HW, Chiou SH and Chu TY: Human umbilical cord mesenchymal stem cells support nontumorigenic expansion of human embryonic stem cells. Cell Transplant 21: 1515-1527, 2012. 
9. Houthuijzen JM, Daenen LG, Roodhart JM and Voest EE: The role of mesenchymal stem cells in anti-cancer drug resistance and tumour progression. Br J Cancer 106: 1901-1906, 2012.

10. Sundararajan V, Sarkar FH and Ramasamy TS: Correction to: The versatile role of exosomes in cancer progression: Diagnostic and therapeutic implications. Cell Oncol (Dordr) 41: 463, 2018.

11. Halkein J, Tabruyn SP, Ricke-Hoch M, Haghikia A, Nguyen NQ, Scherr M, Castermans K, Malvaux L, Lambert V, Thiry M, et al: MicroRNA-146a is a therapeutic target and biomarker for peripartum cardiomyopathy. J Clin Invest 123: 2143-2154, 2013.

12. Roos J, Enlund E, Funcke JB, Tews D, Holzmann K, Debatin KM Wabitsch $\mathrm{M}$ and Fischer-Posovszky P: miR-146a-mediated suppression of the inflammatory response in human adipocytes. Sci Rep 6: 38339, 2016.

13. Yang C, Lim W, Park J, Park S, You S and Song G: Anti-inflammatory effects of mesenchymal stem cell-derived exosomal microRNA-146a-5p and microRNA-548e-5p on human trophoblast cells. Mol Hum Reprod 25: 755-771, 2019.

14. Cheng WC, Liao TT, Lin CC, Yuan LE, Lan HY, Lin HH Teng HW, Chang HC, Lin CH, Yang CY, et al: RAB27B-activated secretion of stem-like tumor exosomes delivers the biomarker microRNA-146a-5p, which promotes tumorigenesis and associates with an immunosuppressive tumor microenvironment in colorectal cancer. Int J Cancer 145: 2209-2224, 2019.

15. Zhang D, Guo H, Feng W and Qiu H: LAMC2 regulated by microRNA-125a-5p accelerates the progression of ovarian cancer via activating p38 MAPK signalling. Life Sci 232: 116648, 2019

16. Hu Z, Cai M, Zhang Y, Tao L and Guo R: miR-29c-3p inhibits autophagy and cisplatin resistance in ovarian cancer by regulating FOXP1/ATG14 pathway. Cell Cycle 19: 193-206, 2020.

17. Livak KJ and Schmittgen TD: Analysis of relative gene expression data using real-time quantitative PCR and the 2(-Delta Delta $\mathrm{C}(\mathrm{T})$ ) method. Methods 25: 402-408, 2001.

18. Dominici M, Le Blanc K, Mueller I, Slaper-Cortenbach I, Marini F, Krause D, Deans R, Keating A, Prockop DJ and Horwitz E: Minimal criteria for defining multipotent mesenchymal stromal cells. The international society for cellular therapy position statement. Cytotherapy 8: 315-317, 2006.

19. Thery C, Witwer KW, Aikawa E, Alcaraz MJ, Anderson JD, Andriantsitohaina R, Antoniou A, Arab T, Archer F, Atkin-Smith GK, et al: Minimal information for studies of extracellular vesicles 2018 (MISEV2018): A position statement of the international society for extracellular vesicles and update of the MISEV2014 guidelines. J Extracell Vesicles 7: 1535750, 2018.

20. Zhang J, Li S, Li L, Li M, Guo C, Yao J and Mi S: Exosome and exosomal microRNA: Trafficking, sorting, and function. Genomics Proteomics Bioinformatics 13: 17-24, 2015.

21. Lai RC, Yeo RW, Tan KH and Lim SK: Exosomes for drug delivery-a novel application for the mesenchymal stem cell. Biotechnol Adv 31: 543-551, 2013.

22. Asare-Werehene M, Nakka K, Reunov A, Chiu CT, Lee WT, Abedini MR, Wang PW, Shieh DB, Dilworth FJ, Carmona E, et al: The exosome-mediated autocrine and paracrine actions of plasma gelsolin in ovarian cancer chemoresistance. Oncogene 39: $1600-1616,2020$

23. Guo H, Ha C, Dong H, Yang Z, Ma Y and Ding Y: Cancer-associated fibroblast-derived exosomal microRNA-98-5p promotes cisplatin resistance in ovarian cancer by targeting CDKN1A. Cancer Cell Int 19: 347, 2019.

24. Hu W, Xu B, Zhang J, Kou C, Liu J, Wang Q and Zhang R: Exosomal miR-146a-5p from treponema pallidum-stimulated macrophages reduces endothelial cells permeability and monocyte transendothelial migration by targeting JAM-C. Exp Cell Res 388: 111823, 2020.
25. McCann JV, Liu A, Musante L, Erdbrugger U, Lannigan J and Dudley AC: A miRNA signature in endothelial cell-derived extracellular vesicles in tumor-bearing mice. Sci Rep 9: 16743, 2019.

26. Cui Y, She K, Tian D, Zhang P and Xin X: miR-146a inhibits proliferation and enhances chemosensitivity in epithelial ovarian cancer via reduction of SOD2. Oncol Res 23: 275-282, 2016.

27. Ding Y, Cao F, Sun H, Wang Y, Liu S, Wu Y, Cui Q, Mei W and Li F: Exosomes derived from human umbilical cord mesenchymal stromal cells deliver exogenous miR-145-5p to inhibit pancreatic ductal adenocarcinoma progression. Cancer Lett 442: 351-361, 2019.

28. Yuan L, Liu Y, Qu Y, Liu L and Li H: Exosomes derived from MicroRNA-148b-3p-overexpressing human umbilical cord mesenchymal stem cells restrain breast cancer progression. Front Oncol 9: 1076, 2019.

29. Kamada M, Koshikawa N, Minegishi T, Kawada C, Karashima T, Shuin T and Seiki M: Urinary laminin-g2 is a novel biomarker of non-muscle invasive urothelial carcinoma. Cancer Sci 106: 1730-1737, 2015.

30. Korbakis D, Dimitromanolakis A, Prassas I, Davis GJ, Barber E, Reckamp KL, Blasutig I and Diamandis EP: Serum LAMC2 enhances the prognostic value of a multi-parametric panel in non-small cell lung cancer. Br J Cancer 113: 484-491, 2015

31. Kosanam H, Prassas I, Chrystoja CC, Soleas I, Chan A, Dimitromanolakis A, Blasutig IM, Ruckert F, Gruetzmann R, Pilarsky C, et al: Laminin, gamma 2 (LAMC2): A promising new putative pancreatic cancer biomarker identified by proteomic analysis of pancreatic adenocarcinoma tissues. Mol Cell Proteomics 12: 2820-2832, 2013.

32. Govaere O, Wouters J, Petz M, Vandewynckel YP, Van den Eynde K, Van den Broeck A, Verhulst S, Dolle L, Gremeaux L, Ceulemans A, et al: Laminin-332 sustains chemoresistance and quiescence as part of the human hepatic cancer stem cell niche. J Hepatol 64: 609-617, 2016.

33. Liang Y, Chen X, Wu Y, Li J, Zhang S, Wang K, Guan X, Yang K and Bai Y: LncRNA CASC9 promotes esophageal squamous cell carcinoma metastasis through upregulating LAMC2 expression by interacting with the CREB-binding protein. Cell Death Differ 25: 1980-1995, 2018

34. Binju M, Amaya-Padilla MA, Wan G, Gunosewoyo H, Suryo Rahmanto Y and Yu Y: Therapeutic inducers of apoptosis in ovarian cancer. Cancers (Basel) 11: 1786, 2019.

35. Zhang W, Zhou Q, Wei Y, Da M, Zhang C, Zhong J, Liu J and Shen J: The exosome-mediated PI3k/Akt/mTOR signaling pathway in cervical cancer. Int J Clin Exp Pathol 12: 2474-2484, 2019.

36. Li H, Xie S, Li H, Zhang R and Zhang H: LncRNA MALAT1 mediates proliferation of LPS treated-articular chondrocytes by targeting the miR-146a-PI3K/Akt/mTOR axis. Life Sci: Aug 28 , 2019 (Epub ahead of print).

This work is licensed under a Creative Commons Attribution-NonCommercial-NoDerivatives 4.0 International (CC BY-NC-ND 4.0) License. 